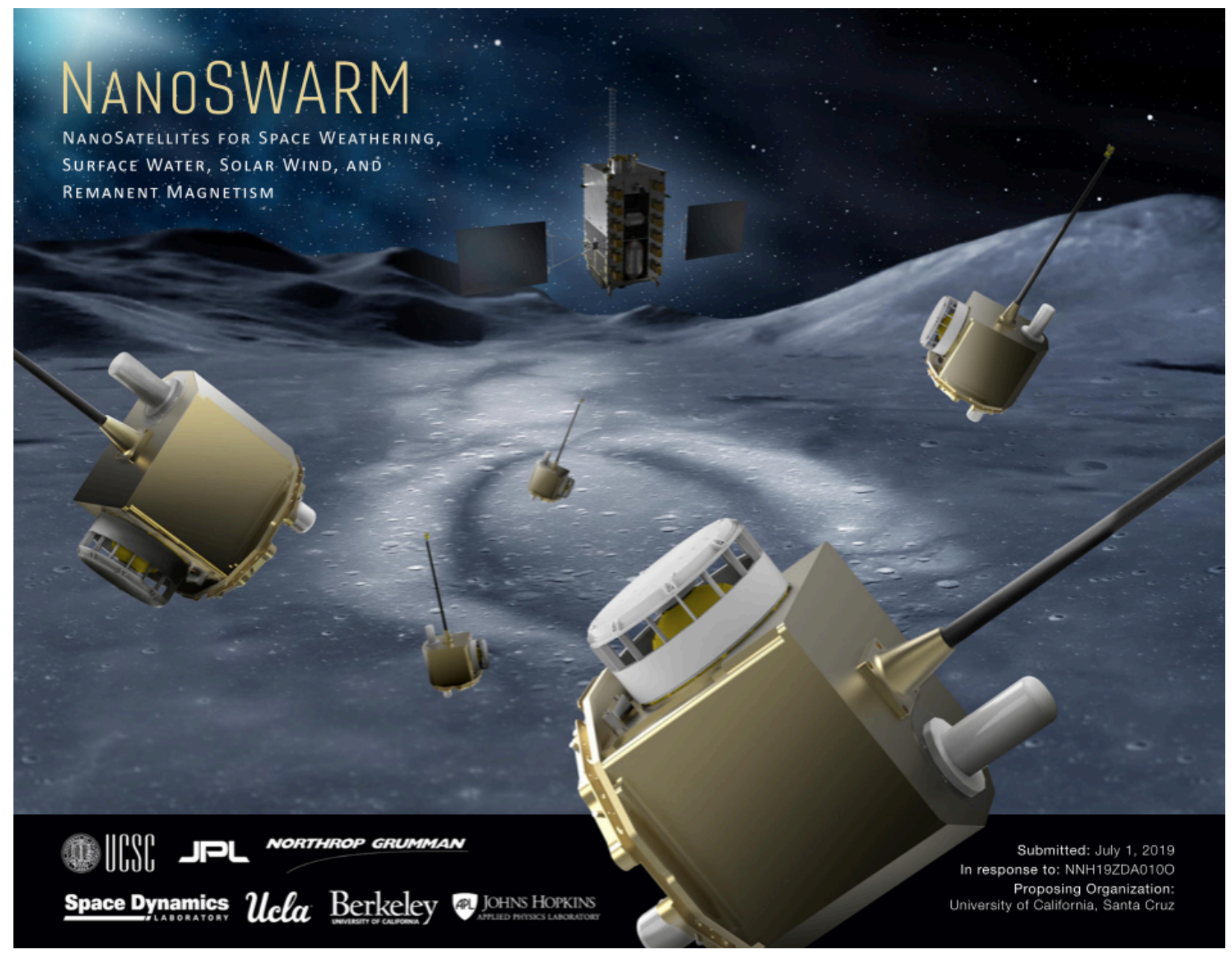

\title{
NanoSWARM: NanoSatellites for Space Weathering, Surface Water, Solar Wind, and Remanent Magnetism
}

Primary author: Ian Garrick-Bethell, UC Santa Cruz, 831-459-1277, igarrick@ucsc.edu

Co-authors: David A. Paige (UCLA), Marcia Burton (JPL), Justin Boland (JPL), Jacob N. H. Abrahams (UC Santa Cruz), Carolyn van der Bogert (U. Münster), Young-Jun Choi (KASI), Jan Deca (University of Colorado), Kerri Donaldson Hanna (U. Central Florida), William M. Farrell (NASA Goddard), Doug Hemingway (Carnegie Institute), Harald Hiesinger (U. Münster), Ho Jin (Kyung Hee University), Brandon C. Johnson (Purdue), Heather Kaluna (U. Hawaii), Megan R. Kelley (UC Santa Cruz), Davin E. Larson (UC Berkeley), David J. Lawrence (JHU Applied Physics Laboratory), Shuai Li (U. Hawaii), Yingjuan Ma (UCLA), Rachel E. Maxwell (UC Santa Cruz), Richard S. Miller (JHU Applied Physics Lab), Carle M. Pieters (Brown University), Andrew R. Poppe (UC Berkeley), Dhananjay Ravat (U. Kentucky), Chris T. Russell (UCLA), Matthew A. Siegler (Planetary Science Institute), Pierre Vernazza (Aix-Marseille U.), and Benjamin P. Weiss (MIT). 
The NanoSWARM mission concept is a detailed investigation of particles and magnetic fields to help characterize the surfaces of airless planetary bodies, their volatile element distributions, and their geophysical histories. NanoSWARM investigates unique regions in the solar system where these processes come together: lunar swirls.

NanoSWARM would be the first demonstration of a planetary-class carrier vehicle equipped with dozens of nanosatellites, each capable of performing measurements either too risky or difficult for the carrier. This architecture greatly facilitates contributed nanosatellites and complete sub-missions from other nations, students, or participating scientists.

NanoSWARM was proposed to the Discovery program in 2019. It was one of 6 missions that received the highest possible ranking of Category 1 , but it did not move forward to Phase $A$.

The commercial space flight community is well on its way toward mass production of small spacecraft. An openness within the planetary community toward building dozens of instruments or spacecraft could open up entirely new regimes of measurement in space.

1. Science overview: NanoSWARM (NanoSatellites for Space Weathering, Surface Water, Solar Wind, And Remanent Magnetism) uses a new type of space mission architecture to address four complementary science goals, becoming, in effect, four missions in one. NanoSWARM launches 19 NanoSatellites (plus spares) to fly just above the surface at five target sites spread across the Moon's near and far sides. This architecture permits a science investigation of substantial breadth and depth, beyond what could be accomplished with a single orbiter, rover, or lander.

NanoSWARM's first goal is to elucidate the mechanisms of space weathering - the alteration of an airless body's optical properties due to solar wind and micrometeoroid bombardment. This ubiquitous process affects spectral data from 0.1-10 microns and, thereby, our ability to determine the compositions of bodies ranging from Mercury to asteroids. Despite decades of study, we do not have a complete model for how space weathering operates. In particular, the relative contributions from micrometeoroids and solar wind flux and the influence of local soil mineralogy on the optical effects are not completely understood. To address this problem, NanoSWARM investigates unique locations on the Moon where solar wind flux is modified while the micrometeoroid flux and mineralogy remain constant: lunar swirls (Fig. 1). The $\mathrm{M}^{3}$ instrument on Chandrayaan-1 as well as Diviner and LAMP on Lunar Reconnaissance Orbiter (LRO) quantified the spectral variations across these features. By performing the first spatially resolved near-surface solar wind flux measurements across multiple swirls with different soil iron contents, NanoSWARM quantitatively resolves how the solar wind affects the optical properties of the Moon and similar silicate bodies.

NanoSWARM's second goal is to constrain how near-surface water forms and is distributed on airless bodies like the Moon, Mercury, and asteroids. Lunar Prospector and Chandrayaan-1 made landmark discoveries of two different water populations: a surficial one that appears at all latitudes and a polar one that may extend to tens of meters below the surface. The connections between these two populations are unknown. NanoSWARM takes advantage of the most recent discovery that lunar magnetic anomalies are correlated with low concentrations of surfacebound $\mathrm{OH} / \mathrm{H}_{2} \mathrm{O}$ ((Li \& Milliken, 2017); Fig. 2). By measuring the in-situ solar wind proton flux at magnetic anomalies, NanoSWARM helps quantify how protons are converted into these products. Furthermore, NanoSWARM makes very low altitude measurements of neutrons above the poles to quantify hydrogen distributions inside craters, but this measurement is not emphasized here. 

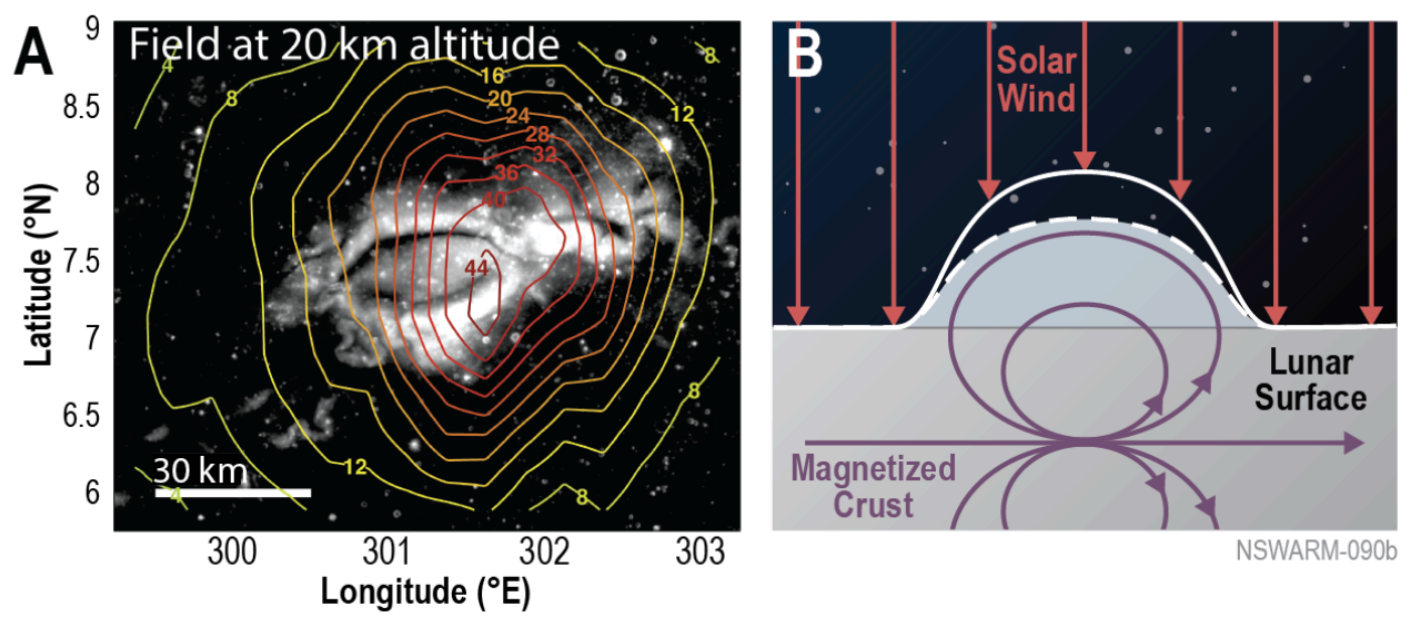

Figure 1. (a) Reiner Gamma (RG) swirl is a lunar magnetic anomaly showing surface albedo variations (black and white features). The image is overlain by contours of magnetic field strength (nT) measured from orbit, which do not correspond to the fine-scale structure in the albedo pattern at this altitude $(20 \mathrm{~km})$. (b) Simplified model illustrating the interaction between the solar wind (a space weathering agent) and a crustal magnetic field, which deflects the solar wind (Hood \& Schubert, 1980).

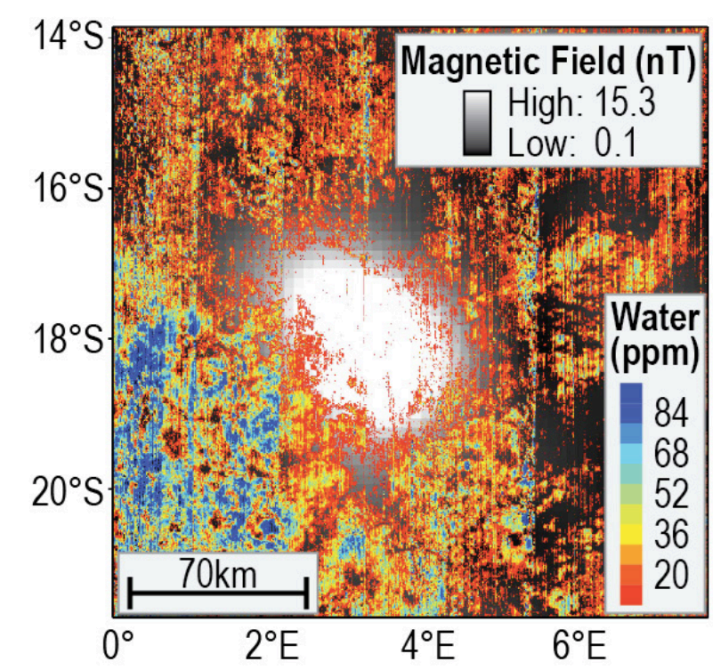

Figure 2. $\mathrm{M}^{3}$ spectroscopic measurements of the water $\left(\mathrm{OH} / \mathrm{H}_{2} \mathrm{O}\right)$ abundance at the Airy magnetic anomaly, overlying the magnetic field measured at 20 km (Li \& Garrick-Bethell, 2019).
NanoSWARM's third goal is to understand how small bodies like the Moon and asteroids have generated dynamos and magnetized their crusts. Dynamos convert the energy of core fluid motions into magnetic fields and can be found in the interiors of bodies throughout the solar system. Understanding how the strength of a planetary body's magnetic field varied over time provides important constraints on its thermal history and extent of differentiation. Studies of lunar samples imply Earthstrength magnetic fields on the Moon (Weiss \& Tikoo, 2014), yet the presence of such fields defies present dynamo theories (Evans et al., 2018). NanoSWARM is uniquely positioned to address this dichotomy because it measures the magnetic field strength above some of the Moon's most magnetized crustal materials at altitudes an order of magnitude lower than those achieved by any previous lunar mission. These measurements constrain the rock magnetization strength, and thereby help estimate the ancient dynamo field strength. NanoSWARM's unique set of measurements will also create a "spectral magnetometer" that enables the estimation of surface crustal magnetic field strength and morphology at more than 100 swirls across the Moon. Together, these measurements will elucidate how the Moon and bodies like it have become magnetized. 


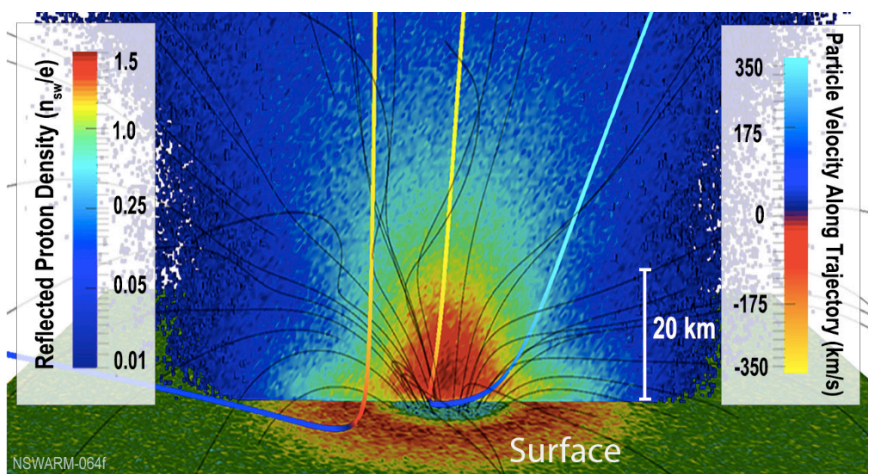

Figure 3. Model of solar wind plasma interaction with a vertical dipole, possibly like the Airy magnetic anomaly, one of NanoSWARM's five target sites (Deca \& Divin, 2016).
NanoSWARM's fourth goal is to investigate the physics of particlefield interactions at the smallest scales. To date, space physics investigations have been largely restricted to planetary and stellar magnetic fields, but there are many contexts where complex small-scale interactions are of interest, such as solar wind interactions with magnetized asteroids (Fatemi \& Poppe, 2018; Omidi et al., 2002) and crustal magnetic fields on Mercury (Johnson et al., 2015) and Mars (Acuña et al., 2001). On the Moon, swirls represent some of the smallest known

obstacles to the solar wind, with some features $\sim 10 \mathrm{~km}$ in size. By measuring the in-situ 3D proton flux and magnetic field at high resolution, NanoSWARM performs the finest-scale study of the smallest magnetospheres in the solar system (Fig. 3).

All of NanoSWARM's individual investigations produce data that complement one another. Magnetic fields influence solar wind fluxes, which in turn influence space weathering rates and surficial $\mathrm{OH} / \mathrm{H}_{2} \mathrm{O}$ production, which may influence the accumulation of water at the poles. The strength of these processes may depend on the body's dynamo history (Garrick-Bethell et al., 2019). In all cases, knowledge of the underlying space physics at both small and large scales is necessary.

\section{Implementation: NanoSWARM} deploys two dozen nanosatellites to fly at kilometer altitudes through the unexplored regions of five of the Moon's magnetic anomalies/swirls. Examples of this operation are shown for the Reiner Gamma anomaly in Figures 4, 5, and 6. Measurements of the plasma flux and magnetic field are performed at very high cadence, at multiple local times of day (Fig. 7). Simultaneous measurements with the carrier vehicle yield additional data at an intermediate altitude $(<$ $10 \mathrm{~km})$, providing a complete threedimensional and time-varying picture of the near-surface plasma flux. These measurements are performed at multiple swirls across the highlands and maria, to determine how soil iron content influences space weathering, and how surface water production varies with magnetic field, surface temperature, sun angle, and other effects.

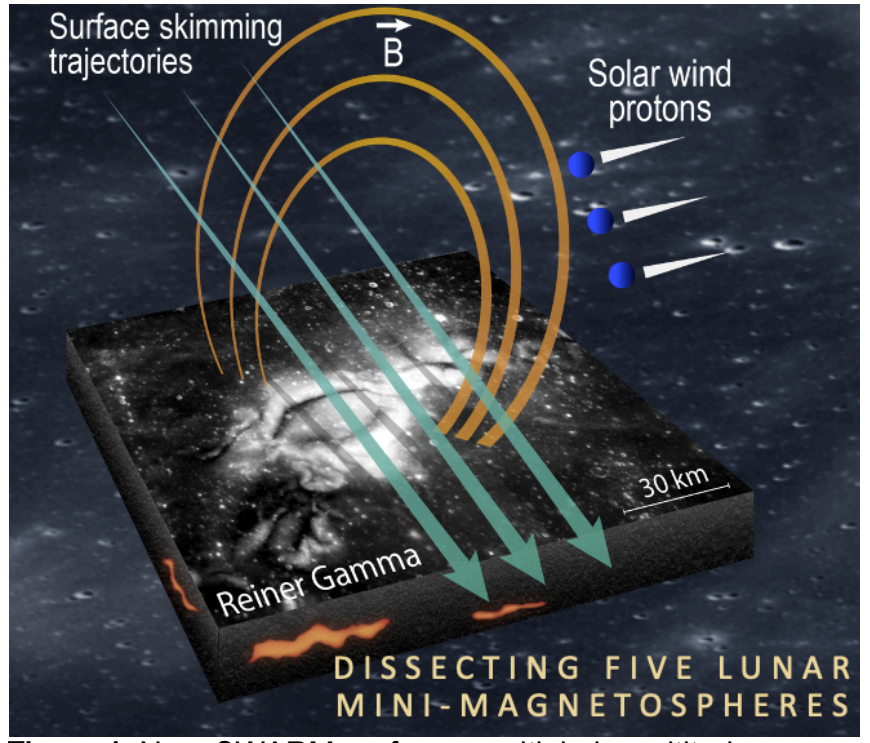

Figure 4. NanoSWARM performs multiple low altitude passes through a set of swirls on surfaces with diverse soil iron concentrations and surface water abundances. Here the Reiner Gamma anomaly is shown. 


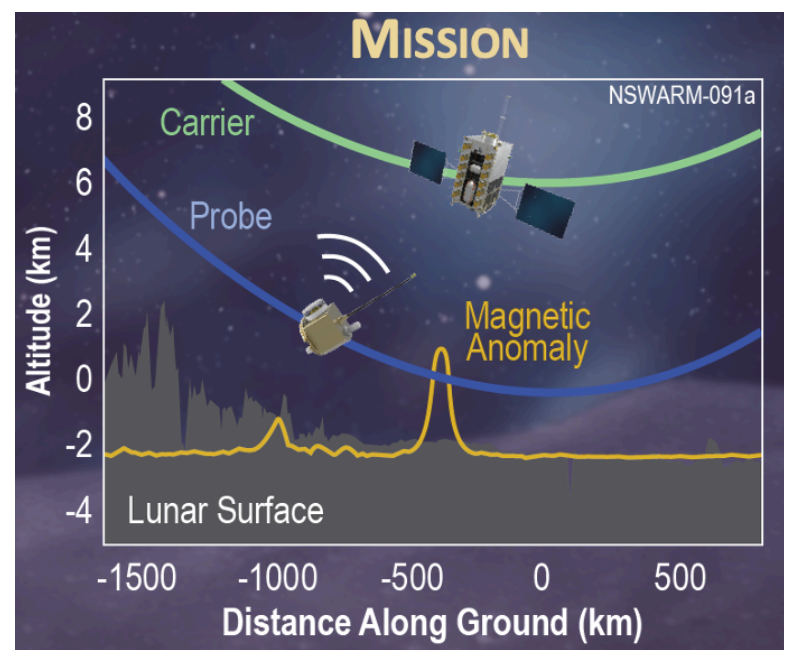

Figure 5. The NanoSWARM carrier vehicle releases multiple probes and receives their data, while performing its own independent measurements at intermediate altitudes.

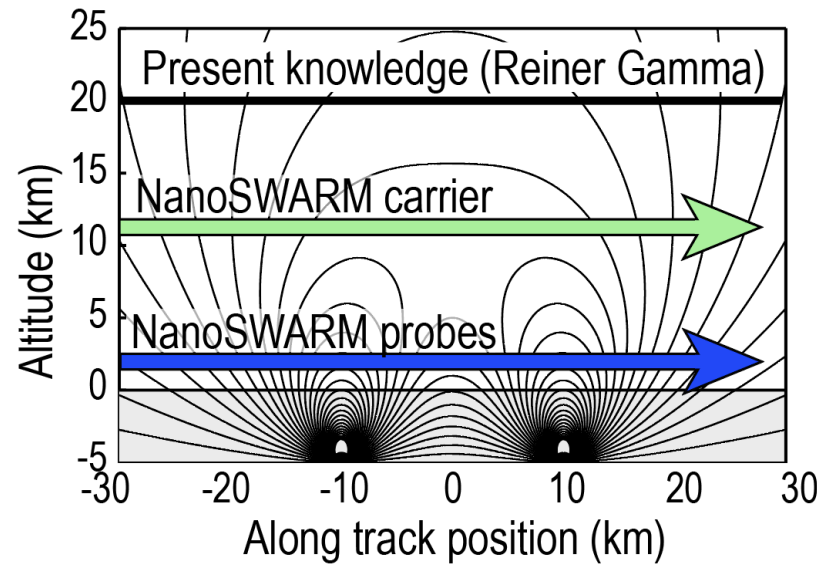

Figure 6. NanoSWARM probes perform their measurements at altitudes that offer an order of magnitude improvement in knowledge of magnetic field structure. Flight through hypothesized cusp structures near the surface permit distinguishing between multiple hypotheses for the origin of the magnetized rock, in addition to determining how plasma flux may be channeled to the surface.

\section{MEASUREMENT PATHS \& SOLAR WIND CONDITIONS (LOCAL TIMES)}

\section{Key: 4 || 0.5-2.5 km altitude probe, simultaneous with $10 \mathrm{~km}$ altitude carrier $10 \mathrm{~km}$ altitude carrier only measurement}
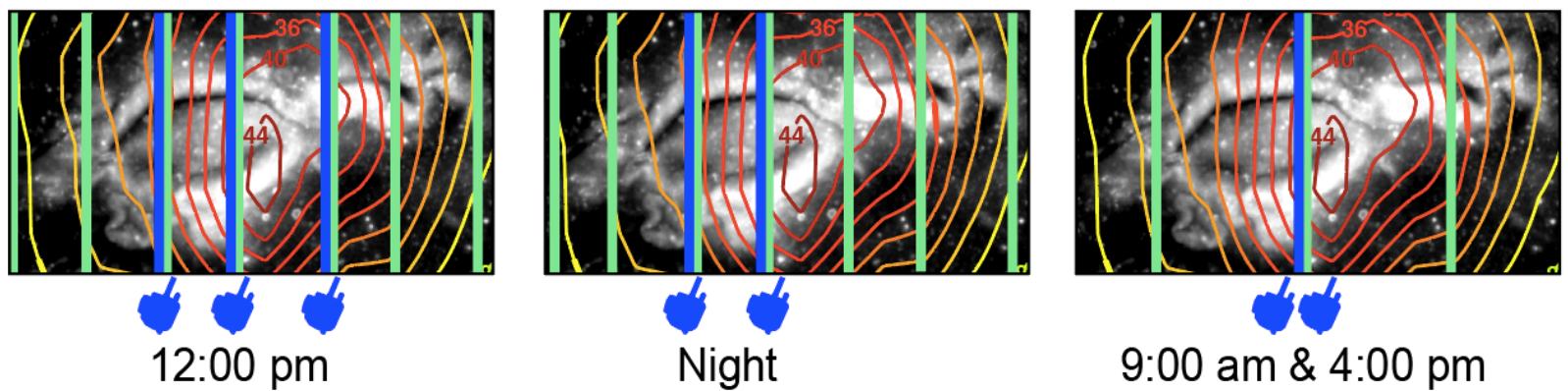

Figure 7. NanoSWARM performs measurements at multiple times of day, at multiple locations within a site (swirl), and at multiple sites across the Moon. The diversity of times, locations, and sites permits a complete investigation of how the solar wind controls surface spectral properties and surface water abundances. At the same time, a diverse set of measurements that constrain the strength of the ancient dynamo field are acquired.

Comparison with lander measurements: Recently, the NASA Commercial Lunar Payload Services Program (CLPS) announced a lander mission to the Reiner Gamma swirl in 2023. This mission has the potential to provide first-of-a-kind full-day plasma and magnetic measurements at single point. However, Reiner Gamma's magnetic field is a complex three-dimensional structure whose interaction with the solar wind plasma extends from the surface to at least $20 \mathrm{~km}$ (e.g. Fig. 3). Furthermore, the main body of the Reiner Gamma surface markings is over $30 \mathrm{~km}$ in extent (Fig. 8), and its patterns vary from excessively dark relative to background surroundings (Garrick-Bethell et al., 2011), to some 
of the brightest terrain on the lunar maria. Finally, it is important to note that Reiner Gamma is not strongly correlated with surface water; several other swirls show much stronger correlations (Li \& Garrick-Bethell, 2019). These other swirls, such as Airy (Fig. 3), offer a better opportunity to determine how the solar wind, temperature, and several other effects combine to generate the local surface water signal (Farrell et al., 2017). Thus, a complete characterization of swirls that addresses all of the planetary phenomena they are associated with (Objectives 1-4) requires: altitude diversity (Fig. 3 and 6), geographic diversity within a single swirl (Fig. 7), and site diversity (e.g. Airy swirl in Fig. 2). A lander measurement at a single point would of course greatly complement such an orbital investigation, and be of high value.

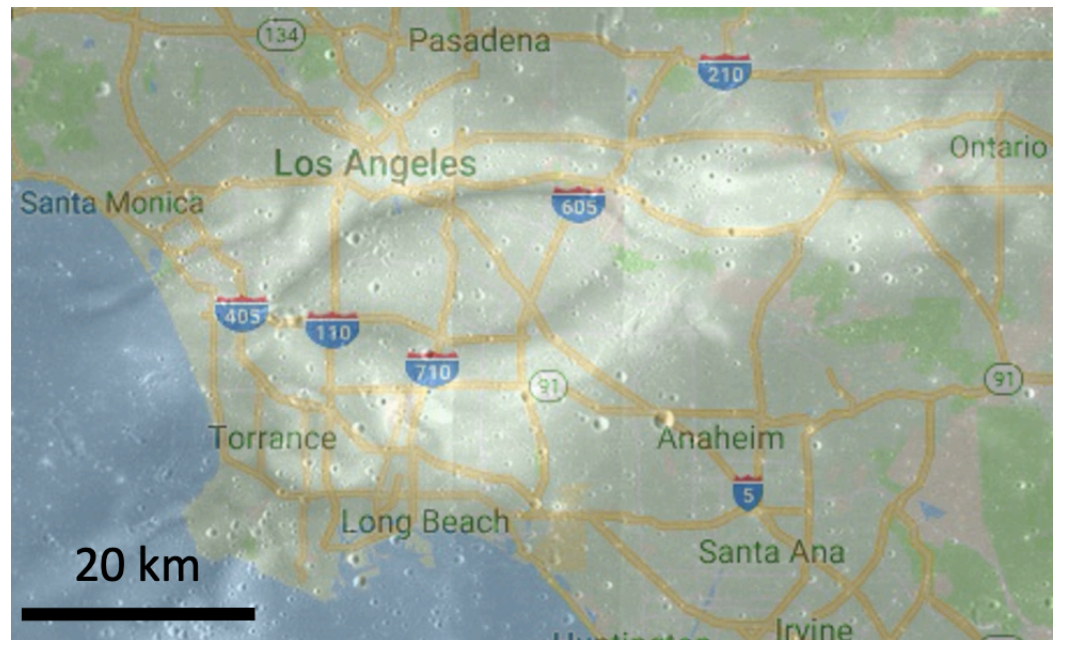

Figure 8. Reiner Gamma has a large and highly variable spectral footprint on the lunar surface, comparable to the size of lower Los Angeles county. Additionally, its 3D field structure extends above the surface. A single point measurement on the surface from the CLPS program would be a very welcome contribution to the study of swirls, but measurements at diverse altitudes, locations, and multiple swirls would completely address all four science objectives of NanoSWARM.

3. Conclusions: An orbital lunar mission that takes advantage of nanosatellite technology and batch production methods could provide numerous insights into important open questions in planetary science. Such a mission would also easily accommodate hosted nanosatellites from other nations, participating scientists, and students, thereby greatly increasing the number of creative science investigations and mission PIs.

NanoSWARM's Category 1 rating in the 2019 Discovery competition illustrates that such a mission is achievable within the Discovery cost cap. Descope of this mission, which is easily achieved by reducing the number of nanosatellites, can also accomplish significant science for a lower cost. 


\section{References:}

Acuña, M. H., et al. (2001), Magnetic field of Mars: Summary of results from the aerobraking and mapping orbits, Journal of Geophysical Research, 106, 23403-23418.

Deca, J., and A. Divin (2016), Reflected Charged Particle Populations around Dipolar Lunar Magnetic Anomalies, The Astrophysical Journal, 829, 60, doi:10.3847/0004-637x/829/2/60.

Evans, A. J., S. M. Tikoo, and J. C. Andrews-Hanna (2018), The Case Against an Early Lunar Dynamo Powered by Core Convection, Geophys. Res. Lett, 45, 98-107, doi:doi.org/10.1002/2017GL075441.

Farrell, W. M., D. M. Hurley, V. J. Esposito, J. L. McLain, and M. I. Zimmerman (2017), The statistical mechanics of solar wind hydroxylation at the Moon, within lunar magnetic anomalies, and at Phobos, Journal of Geophysical Research (Planets), 122, 269-289.

Fatemi, S., and A. R. Poppe (2018), Solar wind plasma interaction with asteroid 16 Psyche: Implication for Formation Theories, Geophys. Res. Lett, 45, 39-48.

Garrick-Bethell, I., J. W. Head, and C. M. Pieters (2011), Spectral properties, magnetic fields, and dust transport at lunar swirls, Icarus, 212(2), 480-492, doi:10.1016/j.icarus.2010.11.036.

Garrick-Bethell, I., A. Poppe, and S. Fatemi (2019), The lunar paleomagnetosphere, Geophysical Research Letters, submitted.

Hood, L. L., and G. Schubert (1980), Lunar magnetic anomalies and surface optical properties, Science, 208, 49-51.

Johnson, C. L., et al. (2015), Low-altitude magnetic field measurements by MESSENGER reveal Mercury's ancient crustal field, Science, 348, 892-895.

Li, S., and I. Garrick-Bethell (2019), Surface water at lunar magnetic anomalies, Geophys. Res. Lett, 46, 14318-14327.

Li, S., and R. E. Milliken (2017), Water on the surface of the Moon as seen by the Moon Mineralogy Mapper: Distribution, abundance, and origins, Science Advances, 3, e1701471.

Omidi, N., X. Blanco-Cano, C. T. Russell, H. Karimabadi, and M. Acuna (2002), Hybrid simulations of solar wind interaction with magnetized asteroids: General characteristics, Journal of Geophysical Research (Space Physics), 107, 1487.

Weiss, B. P., and S. M. Tikoo (2014), The lunar dynamo, Science, 346, 1198. 